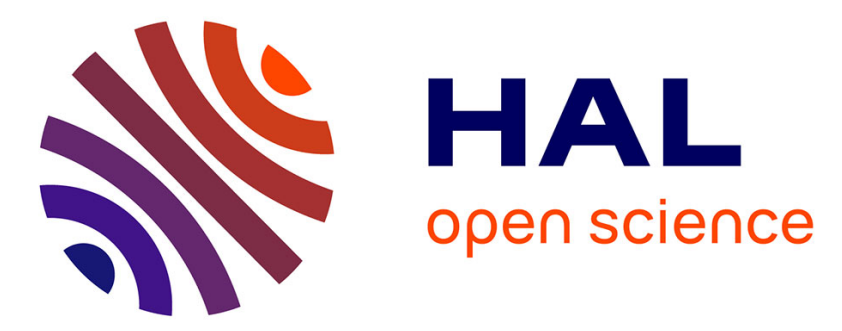

\title{
Développement métalinguistique et apprentissage de la lecture chez les enfants bilingues
}

\author{
Anne-Sophie Besse, Nathalie Marec-Breton, Elisabeth Demont
}

\section{To cite this version:}

Anne-Sophie Besse, Nathalie Marec-Breton, Elisabeth Demont. Développement métalinguistique et apprentissage de la lecture chez les enfants bilingues. Enfance, 2010, 2010 (02), pp.167. 10.4074/S001375451000203X . hal-01893326

\section{HAL Id: hal-01893326 \\ https://hal.science/hal-01893326}

Submitted on 11 Oct 2018

HAL is a multi-disciplinary open access archive for the deposit and dissemination of scientific research documents, whether they are published or not. The documents may come from teaching and research institutions in France or abroad, or from public or private research centers.
L'archive ouverte pluridisciplinaire HAL, est destinée au dépôt et à la diffusion de documents scientifiques de niveau recherche, publiés ou non, émanant des établissements d'enseignement et de recherche français ou étrangers, des laboratoires publics ou privés. 


\title{
Publication 5
}

\author{
Article sous presse \\ revue « Enfance », vol 2, juin 2010.
}

\section{Développement métalinguistique et apprentissage de la lecture chez les enfants bilingues}

\author{
Anne-Sophie Besse ${ }^{1}$, Nathalie Marec-Breton ${ }^{1}$, Elisabeth Demont ${ }^{2}$ \\ ${ }^{1}$ Centre de Recherches en Psychologie, Cognition, Communication. \\ Université Rennes 2
}

${ }^{2}$ Laboratoire de Psychologie des Cognitions

Faculté de Psychologie \& des Sciences de l'Education

Université de Strasbourg 
Résumé : Dans le champ du bilinguisme enfantin et en lien avec l'apprentissage de l'écrit, le développement des compétences métalinguistiques est un domaine de recherche bien développé mais faisant apparaître néanmoins des données contradictoires.

Notre objectif est d'effectuer une analyse critique des recherches conduites ces trente dernières années sur les compétences métaphonologiques, métasyntaxiques, métamorphologiques et l'apprentissage de la lecture en contexte bilingue. La synthèse des données issues de comparaisons entre bilingues et monolingues permettra d'identifier plusieurs facteurs spécifiant les effets du bilinguisme sur le développement métalinguistique et des habiletés en lecture. L'analyse des langues en contact, de leurs relations et de leurs spécificités constitue aussi un champ d'investigation fécond pour aboutir à un point de vue nuancé sur le développement de ces compétences en contexte bilingue.

Mots clés: Bilinguisme enfantin. Développement métalinguistique. Apprentissage de la lecture.

Title: Metalinguistic developpement and reading acquisition in bilingual children

Abstract: In the field of child's bilingualism, researches on metalinguistic development, connected with learning to read, are well developed but show mixed data.

In this paper, a critical analysis is carried out on literature of this last thirty years focused on phonological, syntactical and morphological awareness and reading acquisition in bilingual context. The synthesis of data from bilinguals and monolinguals comparisons allowed to specifying factors underlying various effects of bilingualism on these linguistic awareness and its role on literacy acquisition. The analyses of languages in contact, cross-linguistics relations and features is a productive research field to achieve an accurate point of view on metalinguistic development connected with learning to read in bilingual context.

Key words: Child's bilingualism. Metalinguistic development. Reading acquisition 


\section{Développement métalinguistique et apprentissage de la lecture chez les enfants bilingues}

\section{Introduction}

La compétence ou conscience métalinguistique (metalinguistic awareness en anglais) peut se définir comme « un sous domaine de la métacognition qui concerne le langage et son utilisation, autrement dit comprenant (1) les activités de réflexion sur le langage et son utilisation; (2) les capacités du sujet à contrôler et planifier ses propres processus de traitement linguistique » (Gombert, 1990, p 27). En d'autres termes, les compétences métalinguistiques renvoient à des connaissances explicites relevant d'une activité contrôlée consciemment par le système cognitif, ces connaissances pouvant porter sur différents niveaux du système linguistique : phonologique, syntaxique, morphologique, etc. Du fait de la structure hiérarchique du langage, l'apprenti-lecteur doit prendre conscience que les mots sont segmentables en différentes unités phonologiques (e.g. en syllabes et en phonèmes). C'est en cela qu'intervient, lors de l'apprentissage de la lecture, la conscience phonologique (phonological awareness) ou compétence métaphonologique, définie comme «la capacité à identifier les composants phonologiques des unités linguistiques et à les manipuler de manière délibérée » (Demont \& Gombert, 2007 p. 51). Mais, les systèmes écrits des langues ne se limitent pas à encoder des informations phonologiques. Ils représentent également la structure morphologique des mots. Ainsi, la conscience morphologique (ou compétence métamorphologique), définie par Carlisle (1995) comme une habileté à réfléchir (sur) et à manipuler la structure morphémique des mots contribue à l'apprentissage de l'écrit. En effet, l'analyse en morphèmes (e.g. « chanteurs » est composé de la base « chant », du morphème « eur » et de la marque flexionnelle du pluriel $\left\langle s \|^{1}\right.$ ) permet de soutenir la construction ou l'accès à la signification des mots écrits et la compréhension de la phrase (e.g. Carlisle, 1995 ; Carlisle \& Nomanbhoy, 1993 ; Casalis \& Louis Alexandre, 2000 ; Lyster, 2002). Enfin, la conscience syntaxique ou la compétence métasyntaxique renvoie à la possibilité de raisonner consciemment sur les aspects de la structure des phrases et de contrôler délibérément l'usage des règles de grammaire (Gombert, 1990). Cette compétence intervient lors de l'apprentissage de la lecture en permettant d'utiliser le contexte pour identifier les mots (e.g. Demont \& Gombert, 1996 ; Gaux \& Gombert, 1999 ; Rego \& Bryant, 1993).

\footnotetext{
${ }^{1}$ Dans de nombreuses recherches citées dans cet article (e.g. Armand, 2000 ; Demont, 2001 ; Demont \& Gombert, 1996 ; Demont, Louvet \& Nocus, 2001 ; Gaux \& Gombert, 1999), les connaissances des règles morphoflexionnelles sont davantage considérées comme une compétence métasyntaxique. Par ailleurs, il existe dans certaines langues comme le français trois classes de mots morphologiquement complexes, les mots fléchis (e.g. chats) appartenant à la morphologie flexionnelle ou morpho-syntaxe, les mots dérivés (e.g. chanteur) appartenant à la morphologie dérivationnelle, et les mots composés (e. g. chou-fleur) appartenant à la morphologie compositionnelle
} 
Ainsi, si le rôle que peuvent jouer les compétences métalinguistiques à différents niveaux de l'apprentissage de la lecture est aujourd'hui bien documenté (voir par exemple Demont \& Gombert, 2007 ; Kuo \& Anderson, 2006), les recherches sur le développement de ces compétences et leur relation avec l'apprentissage de la lecture chez les enfants bilingues permettent plus difficilement d'aboutir à un point de vue clair et univoque sur les spécificités bilingues. Pourtant, un bref aperçu des situations linguistiques des pays du monde et des flux migratoires permet de remarquer que même chez les enfants, le monolinguisme ou l'utilisation d'une seule langue est loin d'être la norme. Le contact de deux langues ou plus, que ce soit dans le domaine du langage oral ou écrit, prend des formes multiples, les langues en contact, le contexte de leur acquisition ou de leur apprentissage, le statut et le niveau de maîtrise étant souvent très différents. Ainsi, l'acception de la notion de bilinguisme varie beaucoup selon les auteurs. Une clarification terminologique s'avère donc nécessaire avant de présenter les recherches étudiant, en contexte bilingue, le développement métalinguistique en lien avec l'apprentissage de la lecture. Cette présentation se focalisera en priorité sur le schéma méthodologique d'une comparaison enfants monolingues / enfants bilingues afin de mieux appréhender les spécificités de ces derniers. Les résultats de ces recherches sont souvent fort différents voire même contradictoires, les unes arguant un avantage métalinguistique des bilingues par rapport aux monolingues, les autres n'ayant pas observé cette supériorité ou révélant même certaines difficultés. Une analyse critique de ces travaux permettra de comprendre ces différences et d'envisager, pour mieux les dépasser, des objectifs de recherches plus axés vers une perspective interactionnelle, tenant compte des spécificités des langues en contact.

\section{Précisions terminologiques : du bilinguisme aux degrés de bilinguisme}

Le sens populaire donné au bilinguisme, rejoint les définitions des dictionnaires courants et de plusieurs auteurs (Bloomfield, 1933 ; Halliday, McKintosh \& Strevens, 1970² ; Marouzeau, 1961 ${ }^{3}$; Thiéry, $1976^{4}$ ) qui envisagent le bilinguisme comme la maitrise parfaite de deux langues. Dans cette acception, les bilingues utilisent couramment et indifféremment deux langues, sans aptitude plus marquée pour l'une que pour l'autre. Bloomfield $\left(1933^{4}, 1958^{3}\right)$ considère même que l'aisance des bilingues doit être comparable à celle des locuteurs de langue maternelle. Ainsi, il est affirmé, plus ou moins explicitement, un équilibre entre les langues connues des bilingues, considérant, pour ainsi dire, le bilinguisme comme un double monolinguisme. Cependant, pour beaucoup d'autres auteurs, ces définitions et conceptions du bilinguisme «vrai » ou «parfait» sont trop restrictives pour appréhender la complexité du phénomène de contact des langues et se révèlent donc peu adaptées voire illusoires (e.g. Mackey, 1976 ; Perregaux, 1994 ; Titone, 1972). Il est en effet aisé de

\footnotetext{
${ }^{2}$ Cités par Perregaux (1994).

${ }^{3}$ Cité par Titone (1972).

${ }^{4}$ Cité par Bruyninckx \& Harmegnies (1993).
} 
rétorquer d'une part, que le degré de perfection dans la maîtrise des langues, que ce soit chez les monolingues ou les bilingues, est difficile à évaluer car extrêmement variable, et d'autre part, que la parité absolue dans le contrôle des deux langues est très rare.

Ainsi, la conception la plus souvent adoptée est celle qui considère le bilinguisme non pas comme un « tout ou rien » mais comme un processus individuel de développement de connaissances linguistiques et langagières, mettant en jeu deux codes distincts et coexistant à des degrés variés, d'une compétence minimale à une maîtrise complète. Ce processus s'élabore sur des éléments culturels, sociologiques et psychologiques et permet une adaptation permanente de l'activité langagière à la situation dans laquelle elle se déroule.

Cette conception souligne la complexité des phénomènes de contact de langues et l'existence de multiples facteurs pour expliquer la diversité des situations bilingues (Bialystok, 2002 ; Mackey, 1976). Mackey (1976) dégage plusieurs variables affectant le degré d'aptitude, en compréhension, expression, lecture et écriture, atteint au niveau phonologique ou graphique, grammatical, lexical, sémantique et stylistique par exemple, la fonction interne et externe des langues (la représentation identitaire, le rôle communicationnel, la conception socioculturelle de la langue), l'influence d'une langue sur l'autre, l'alternance dans l'utilisation des deux langues, etc. D'autres facteurs comme l'âge, les aptitudes générales et intellectuelles des sujets, les langues impliquées, le niveau socioéconomique sont également à l'origine de variations importantes dans les formes prises par le bilinguisme.

L'existence de ces variations est souvent évoquée dans la littérature. On retrouve ainsi régulièrement l'ajout d'un qualificatif précisant à quel type de bilinguisme il est fait référence (pour une revue voir par exemple Bruyninckx \& Harmegnies, 1993; Cummins \& Swain, 1986; Lucchini, 2002). Ces distinctions portent généralement sur les modes d'acquisition du bilinguisme (e.g. bilinguisme scolaire vs. naturel), les conséquences de l'acquisition des langues (e.g. bilinguisme additif vs. soustractif ${ }^{5}$ ) ou encore sur les aspects temporels de l'acquisition. On retrouve ainsi la distinction entre bilinguisme simultané et consécutif ou entre bilinguisme précoce et tardif (à partir du moment où la langue seconde n'intervient dans l'environnement enfantin qu'après l'âge de 3 ou 4 ans $^{6}$ ). La durée qui sépare l'apprentissage des deux langues a en effet un impact important sur l'acquisition du système linguistique (Berthoud, 1982).

Enfin, les variations en terme de maîtrise des deux langues en contact renvoie souvent à l'existence de différents degrés de bilinguisme et à la relation pouvant être faite entre l'apprentissage d'une langue seconde et le bilinguisme. Bialystok, McBride-Chang et Luk (2005b) considèrent ainsi que « the differences in degree of bilingualism is captured by a terminological distinction between

\footnotetext{
${ }^{5}$ Dans le bilinguisme additif les deux langues se maintiennent et se cumulent, alors que dans le bilinguisme soustractif une des deux langues (en général, la langue première) cède la place à l'autre.

${ }^{6}$ D’après McLaughlin (1984) cité par Perregaux (1994).
} 
bilingualism, referring to the control over two linguistic systems, and second-language acquisition, referring to the process of appending a new language to an established system » ( $\mathrm{p} 581)$. Cependant, pour ces auteurs, il n'existe pas de limites claires entre ces deux termes si bien que le passage de l'apprentissage d'une langue seconde au bilinguisme doit être considéré comme un continuum et non pas comme un changement catégoriel. Cette conception rejoint celle de Lucchini qui suggère que « la présence conjointe d'une langue d'origine et d'une langue seconde introduit l'enfant dans le monde du bilinguisme» (Lucchini, 2002, p. 19) et permet d'envisager le bilinguisme selon une acception assez large englobant l'ensemble des degrés de compétences en langue (Bialystok et al., 2005b).

Dans cette perspective, le bilinguisme constitue un phénomène de contact entre deux langues, et d'usage alterné de ces dernières et concerne notamment :

- les enfants issus de foyer bilingue, appartenant à une communauté bilingue, ou encore, immergées régulièrement dans différentes communautés linguistiques.

- les enfants immigrés ou d'origine immigrée, dont la langue d'origine (employée plus ou moins strictement dans le cercle familial) n'est pas la langue du pays de résidence.

- les enfants scolarisés dans des établissements bilingues ou internationaux.

Ainsi, bien que ces enfants soient dans des situations différentes et puissent avoir des compétences linguistiques variables, les recherches citées ultérieurement et portant sur ces populations font toutes référence au bilinguisme. La simultanéité dans l'acquisition des langues en contact ainsi que l'équivalence de leur maîtrise étant rares, il est souvent fait référence, dans le développement suivant, à la langue première et seconde de l'enfant de façon à rendre compte de ce déséquilibre.

\section{Les compétences métalinguistiques des enfants bilingues}

Une part non négligeable de recherches axées sur le bilinguisme enfantin a étudié l'impact du contact des langues sur le développement cognitif en général et sur les compétences métalinguistiques, en particulier.

Certains auteurs ont avancé que la confrontation et l'emploi de plusieurs systèmes linguistiques, engendre une distanciation précoce entre les aspects formels et sémantiques du code, et donc de meilleures capacités d'abstraction et de manipulation conceptuelle ainsi qu'une facilité à porter attention aux aspects formels du langage (e.g. MacLaughlin, 1991; Peal \& Lambert, 1962 ; Vygotsky, 1934). Il a ainsi été observé que les enfants bilingues perçoivent précocement la relation arbitraire entre les mots et leur signification (e.g. Cummins, 1978 ; Ianco-Worall, 1972). D'autres recherches ont également attesté la plus grande flexibilité des capacités cognitives des bilingues (e.g. BenZeev, 1977 ; Bialystok, 2005, 2007 ; Pearl \& Lambert, 1962). 
Plus spécifiquement, Vygotsky (1934) explique que la possibilité d'exprimer la même pensée dans deux langues différentes rend l'enfant capable de voir sa langue comme un système particulier parmi d'autres, ce qui l'amène à prendre conscience des opérations linguistiques propres aux langues. En d'autres termes, l'avantage des bilingues concernerait plus particulièrement le domaine des compétences métalinguistiques, dans le sens où les formes linguistiques se révèlent plus saillantes donc plus facilement accessibles à la conscience.

Bialystok (1991, 1993, 1999, 2001) considère pour sa part l'avantage bilingue en se basant sur la distinction de deux composantes :

- la composante d'analyse, renvoyant aux capacités à se représenter de façon de plus en plus explicite et abstraite la structure linguistique des langues.

- la composante de contrôle, renvoyant aux compétences à porter attention aux aspects spécifiques de ces représentations notamment en situation ambiguës ou trompeuses.

Pour Bialystok, les changements des représentations mentales sont attribuables au développement de ces deux composantes, développement qui permet de comprendre la progression des simples compétences conversationnelles à un usage intentionnel des règles linguistiques lors de la lecture et la résolution de problèmes métalinguistiques. A l'issue de plusieurs expérimentations (Bialystok, 1986 cf. partie 2.2), Bialystok avance que l'avantage bilingue concerne principalement les procédures de contrôle mais pas la dimension d'analyse. Les bilingues seraient ainsi moins distraits par les dimensions non pertinentes lors de la résolution de certaines tâches linguistiques mais aussi non linguistiques (par exemple, une tâche de classification de cartes avec changement de catégories ; Bialystok, 1999), capacités dépendantes des processus de contrôle inhibiteur relevant des fonctions exécutives (Bialystok, 2007). La distinction entre analyse et contrôle, faite par Bialystok, a l'avantage de décrire les processus fondamentaux que les bilingues mettent en place aisément et d'établir les relations entre développement cognitif et métalinguistique. Cependant, cette distinction n'est pas toujours exploitée par les autres chercheurs.

Il faut enfin souligner que ces visions positives du bilinguisme n'ont pas toujours été adoptées (e.g. Palmer, 1972) et certaines recherches n'ont d'ailleurs pas permis d'aboutir à de telles conclusions. Nous présenterons donc tout d'abord quelques données empiriques vérifiant cet avantage bilingue sur certaines dimensions des habiletés métalinguistiques associées à la lecture. Les recherches qui ne sont pas parvenues à démontrer cette facilitation seront ensuite développées. Lorsque ces recherches apportent des résultats sur le développement des compétences en lecture ou encore lorsqu'une distinction entre différents groupes de bilingues est effectuée, les données et conclusions seront également présentées car elles apportent un éclairage non négligeable à la problématique ciblée dans cette revue de question. 


\subsection{Le bilinguisme facilite le développement métalinguistique}

Dans une étude suisse réalisée auprès d'enfants de familles immigrées de tous horizons ayant appris leur langue première jusqu'à 4 ans et apprenant le français depuis leur entrée à l'école, Perregaux (1994, 1995) montre que, malgré leurs difficultés en compréhension et production lexicale en français, les bilingues prélecteurs de grande section de maternelle réussissent aussi bien les tâches métaphonologiques de segmentation phonémique que les monolingues, et même mieux celles portant sur les pseudo-mots. En fin de $1^{\mathrm{e}}$ année, bilingues et monolingues présentent le même niveau en lecture de mots. Grâce à leurs habiletés métalinguistiques, les bilingues ont pu faire face à leur manque de maîtrise orale.

De même, Eviatar et Ibrahim (2001) observent que les bilingues russe-hébreu (parlant russe avec leur famille et hébreu à l'école) en dernière année de maternelle obtiennent de meilleures performances que les monolingues de langue hébreu aux tâches métaphonémiques (i.e. répéter le premier ou dernier phonème d'un mot). Cette différence n'est plus significative en $1^{\mathrm{e}}$ année, mais pour ce niveau scolaire, les résultats à une tâche de suppression de phonème ou de syllabe réitèrent la supériorité des bilingues par rapport aux monolingues. Ces derniers résultats sont également retrouvés par Ibrahim, Eviatar et Aharon-Peretz (2007), auprès d'une même population de $1^{\mathrm{e}}$ année. Comme dans l'étude de Perregaux (1994, 1995), ces auteurs remarquent que les connaissances lexicales en hébreu sont plus faibles chez les bilingues mais pas les performances en lecture de mots non vocalisés et de textes vocalisés ${ }^{7}$. Ces habiletés sont expliquées, chez les bilingues comme les monolingues, par les compétences métaphonologiques ainsi que, mais chez les monolingues uniquement, par les compétences en vocabulaire. Ces résultats suggèrent, encore une fois, que la conscience phonologique permet aux bilingues de compenser leur manque de vocabulaire lors de la lecture.

Campbell et Sais (1995) corroborent l'avantage bilingue dans le domaine métaphonologique avec des enfants bilingues italien-anglais scolarisés dans une école maternelle bilingue. En anglais, les jeunes bilingues sont plus performants que les monolingues à détecter les intrus phonologiques (i.e. le mot, parmi trois, ne commençant pas par le même son) et à supprimer la première syllabe de pseudo-mots.

Dans une recherche plus récente et auprès d'enfants bilingues français - occitan plus âgés suivant un programme d'éducation bilingue depuis l'école maternelle, Laurent et Martinot (2009) étudient les compétences métaphonologiques en administrant des tâches de suppression et de permutation de syllabes et de phonèmes. En $3^{\mathrm{e}}$ année, aucune différence entre bilingues et monolingues n'est significative, mais un an plus tard, les enfants bilingues sont plus performants que les monolingues

\footnotetext{
${ }^{7}$ Rappelons qu'en hébreu, deux formes d'écriture coexistent : une forme vocalisée marquant les voyelles diacritiques et une forme non vocalisée où les voyelles diacritiques ne sont pas écrites.
} 
aux tâches de permutation de syllabes et de permutation de phonèmes de pseudo-mots. En $5^{\mathrm{e}}$ année, l'avantage des bilingues concerne non seulement la permutation de syllabes et de phonèmes mais également la suppression de phonème médian (de mots et de pseudo-mots). Les auteurs interprètent ces résultats comme un élément en faveur de l'hypothèse du niveau seuil postulée par Cummins (1976, 1979, 1991). Selon cette hypothèse, le bilinguisme influencerait positivement le développement métalinguistique lorsqu'un seuil minimal de compétence est atteint dans les deux langues.

L'avantage bilingue a également été observé dans le domaine métasyntaxique et métamorphologique. Concernant la conscience syntaxique, Bialystok (1986) a montré qu'à 5, 7 et 9 ans, les bilingues d'origines immigrées diverses parlant une langue dans leur milieu familial et l'anglais à l'école parviennent mieux que les monolingues (appariés en âge et en compétence orale en anglais) à juger de la grammaticalité de phrases correctes mais sémantiquement anormales (phrase asémantiques), items évaluant les habiletés à porter attention à la forme syntaxique plutôt qu'à la signification. Cependant, elle n'obtient pas cette supériorité des bilingues pour le jugement de phrases agrammaticales mais sémantiquement correctes ni pour le jugement et la correction de phrases agrammaticale et asémantiques, mesurant pour leur part les capacités d'analyse. Cromdal (1999), réplique, en Suédois et au niveau préscolaire (enfants ayant entre 6 et 7 ans), une partie des résultats de Bialystok (1986) à l'aide d'épreuves similaires en comparant les résultats de monolingues, d'enfants partiellement bilingues (i.e. dont les compétences en vocabulaire en suédois sont plus faibles qu'en anglais et inférieures à celles des monolingues) et d'enfants hautement bilingues (i.e. dont les connaissances lexicales en suédois sont équivalentes à celles des monolingues). Comme dans l'étude de Bialystok (1986), les deux groupes bilingues, obtiennent des scores supérieurs aux monolingues lorsqu'il s'agit de juger la grammaticalité de phrases asémantiques, les résultats correspondant à des scores planchers pour ce qui concerne leur correction. Mais pour les phrases sémantiques, le jugement et la correction sont seulement supérieurs pour les enfants hautement bilingues. Bialystok (1988) avait d'ailleurs obtenu des résultats similaires en correction d'agrammaticalité avec des enfants anglais - français partiellement ou hautement bilingues de $1^{\mathrm{e}}$ année (les premiers sont des enfants anglophones suivant un programme scolaire d'immersion en français et les seconds sont scolarisés en école française et parlent le français et l'anglais dans le cadre familial). Le bilinguisme peut ainsi, dans certains cas, favoriser également le développement des compétences d'analyse métasyntaxique.

Dans une étude comparative entre des enfants hautement bilingues espagnol-anglais de niveau préscolaire, maternelle et $1^{\mathrm{e}}$ année et des monolingues hispanophones et anglophones, Galambos et Goldin-Meadow (1990) avaient également détecté une supériorité bilingue aux tâches de jugement 
de grammaticalité (de phrases sémantiquement acceptables ${ }^{8}$ ) en espagnol, langue dans laquelle ils sont aussi performants que le groupe monolingue hispanophone mais pas en anglais, langue dans laquelle ils sont moins performants que leurs pairs monolingues anglophones. Par ailleurs, en anglais comme en espagnol, les bilingues les plus jeunes ont de meilleures performances en correction. Ils produisent en outre plus de corrections basées sur la forme grammaticale que les monolingues dont les corrections aboutissent, à ce niveau, à une modification sémantique (e.g. the doctor is it selling corrigé en the doctor is checking). Enfin, lorsqu'il s'agit d'expliciter les erreurs grammaticales, les bilingues obtiennent les mêmes résultats que les monolingues avec toutefois moins d'explicitations axées sur le contenu sémantique. Ainsi, les auteurs en concluent que les bilingues sont plus précocement en mesure de se centrer sur la forme pour détecter et corriger les erreurs grammaticales. Cet avantage n'est pas visible lorsqu'il s'agit d'expliciter la règle affectée par l'erreur car cette tâche est encore trop complexe pour des enfants aussi jeunes.

Dans le même ordre d'idées, Demont (2001) observe, dans une étude menée en Alsace sur le bilinguisme français-allemand, que les enfants de $1^{\mathrm{e}}$ année scolarisés sur un site bilingue, témoignent, en français, d'une conscience syntaxique plus élevée (évaluée par des épreuves de jugement et de correction de phrases sémantiques ou asémantiques dont les anomalies concernent l'ordre des mots et les affixes flexionnels) et d'une efficience supérieure dans la reconnaissance des mots comparativement à leurs pairs scolarisés dans une classe traditionnelle monolingue. Les analyses de régression rendent également compte du fait qu'au début de l'année scolaire, la contribution de l'apprentissage de l'allemand à la reconnaissance de mots écrits apparaît médiatisée par la conscience syntaxique alors que cette contribution devient indépendante des capacités métasyntaxiques en fin d'année scolaire. Demont (2001) en conclut que le bilinguisme français allemand a une incidence sur le niveau de lecture en français en facilitant, en début d'apprentissage, la prise de conscience de la structure syntaxique du langage.

Concernant, la compétence métamorphologique, Campbell et Sais (1995) ont enfin observé, outre la supériorité des performances aux tâches métaphonologiques précédemment citées, que les bilingues italiens - anglais scolarisés en école maternelle étaient plus performants à supprimer un morphème dans des mots morphologiquement composés (e.g. répéter rain dans rainbow) comparés à leur pairs monolingues. L'ensemble des résultats de cette étude indique ainsi que l'avantage des bilingues concerne la conscience phonologique mais aussi la conscience morphologique.

Ces différentes recherches ont ainsi permis d'observer une supériorité des bilingues sur certaines dimensions métalinguistiques, notamment, phonologique, syntaxique et morphologique. Les résultats de Perregaux (1994, 1995) et de Demont (2001) suggèrent en outre que certaines de ces

\footnotetext{
${ }^{8}$ Ces items renvoient, selon Bialystok $(1986,1988,1991)$ à la dimension d'analyse.
} 
compétences, en l'occurrence les compétences métaphonologiques et métasyntaxiques jouent un rôle sur la reconnaissance des mots écrits, dont le niveau équivaut ou peut même être supérieur à celle des monolingues. Cependant, cet avantage bilingue concernant les habiletés métalinguistiques ne semble toutefois pas être attesté sur l'ensemble des tâches utilisées et à tous les âges (e.g. Eviatar \& Ibrahim, 2000 ; Galambos \& Goldin-Meadow, 1990 ; Laurent \& Martinot, 2009). Les données présentées dans le paragraphe suivant renforcent ce constat et tendent même à montrer un déficit des bilingues dans certains domaines métalinguistiques.

\subsection{Le bilinguisme ne facilite pas le développement métalinguistique}

Concernant la compétence métaphonologique, la recherche de Demont (2001), citée précédemment n'était pas parvenue à voir apparaître de différences significatives entre les deux groupes bilingues /monolingues aux tâches de suppression de phonème. Pour expliquer ce résultat, Demont (2001) suggère que la conscience phonologique est liée, à ce niveau, à l'enseignement des correspondances graphophonologiques, enseignement réalisé dans toutes les classes.

Plusieurs recherches n'ont d'ailleurs pas observé de différences significatives entre bilingues et monolingues sur la dimension métaphonologique et ont même détecté une insuffisance des bilingues dans le domaine métasyntaxique.

Par exemple, Demont, Louvet et Nocus (2001), à l'aide d'instruments de mesure similaires et sur une population pourtant proche voire identique à celle de Demont (2001), n'obtiennent pas les mêmes résultats que cette dernière. En effet, les enfants issus d'écoles bilingues français-allemand à parité horaire (comme dans l'étude de Demont, 2001), n'ont pas de meilleurs résultats que les monolingues ni en suppression phonémique, ni en jugement et correction de phrases agrammaticales, ni même en lecture de mots, de pseudo-mots et en compréhension de phrases. Par ailleurs, les enfants scolarisés en $1^{\mathrm{e}}$ année d'école internationale, recevant 5 ou 6 heures d'enseignement de l'allemand, commettent plus d'erreurs de jugement sur la grammaticalité de phrases en français construites selon la structure syntaxique de l'allemand. Les auteurs interprètent ces résultats en évoquant une possible interférence entre les connaissance de la structure syntaxique des deux langues et de l'existence d'un niveau seuil de compétences, non encore atteint par les enfants de $1^{\mathrm{e}}$ année, pour pouvoir tirer profit de l'apprentissage d'une langue seconde.

Toujours auprès d'enfants de $1^{\mathrm{e}}$ année, Armand (2000) compare les compétences métalinguistiques d'enfants francophones monolingues et d'enfants immigrés au Québec ou d'origine immigrée, le niveau de lecture en français des deux groupes étant équivalent. Elle obtient une infériorité des bilingues dans la réussite aux épreuves de jugement et de correction d'agrammaticalité (portant sur l'ordre des mots et les morphèmes flexionnels) mais pas aux épreuves métaphonologiques (détection de rime et suppression de phonème). En outre, les bilingues ont des compétences 
langagières orales plus faibles que les monolingues (test de vocabulaire et de compréhension orale) que l'auteur explique par la moindre expérience linguistique. D'après les analyses de corrélation et de régression, le traitement des aspects syntaxiques en français ne peut se manifester pleinement à cause de ces lacunes en compétences langagières. Enfin, elle en conclut un rôle fondamental des capacités métaphonologiques. Du fait de leur prédictibilité sur la reconnaissance des mots écrits, elles tiennent une place primordiale dans l'apprentissage de la lecture en langue seconde et permettent de compenser les faiblesses en termes d'habiletés langagières orales et de compétences métasyntaxiques.

Par ailleurs, bien que les recherches de Chiappe et Siegel (1999), Da Fontoura et Siegel (1995) et Lesaux et Siegel (2003) aient pour objectif d'examiner si l'acquisition de la lecture est déterminée par les mêmes processus et habiletés en langue première et seconde, voire même de rendre compte des effets positifs du bilinguisme sur cette acquisition, leurs résultats échouent à mettre en évidence une supériorité bilingue à plusieurs tâches métalinguistiques.

Par exemple, Chiappe et Siegel (1999) ont réalisé une recherche en langue anglaise auprès d'enfants locuteurs punjabi de $1^{\mathrm{e}}$ année, exposés à l'anglais depuis deux ans. Si les scores à plusieurs tâches métaphonologiques (identification, localisation, suppression et substitution phonémique) sont équivalents pour les deux groupes, les élèves bilingues obtiennent des scores plus faibles que leurs pairs monolingues anglophones à une épreuve métasyntaxique de complètement de phrase ou closure (e.g. Jane __ her sister ran up the hill). Toujours en anglais et à l'aide du même outil d'évaluation, Da Fontoura et Siegel (1995) ont également observé cette faiblesse dans le domaine syntaxique avec des enfants d'origine portugaise plus âgés (en $4^{\mathrm{e}}, 5^{\mathrm{e}}$ et $6^{\mathrm{e}}$ année) suivant un programme d'apprentissage de la lecture en langue maternelle. Pourtant, comme dans l'étude de Chiappe et Siegel (1999), les habiletés de reconnaissance de mots en anglais, atteignent un niveau d'efficience similaire à ceux des monolingues. Les enfants bilingues en difficulté d'apprentissage ont même des scores plus élevés en lecture de pseudo-mots que les lecteurs monolingues en difficulté. Les auteurs en concluent que le bilinguisme n'a pas forcément de conséquence négative sur le développement des habiletés en lecture. Leur faiblesse dans le domaine des habiletés métasyntaxiques serait essentiellement due à une exposition insuffisante à la complexité syntaxique de l'anglais pour atteindre le niveau d'efficience des monolingues. Toutefois, les auteurs observent que la conscience syntaxique développée spécifiquement en anglais entretient un lien corrélationnel avec la lecture en anglais.

Dans le même ordre d'idées, Lesaux et Siegel (2003) ont mené une étude longitudinale sur l'anglais langue seconde en suivant des enfants immigrés ou d'origine immigrée de différentes langues premières, de la dernière année de maternelle à la $2^{\mathrm{e}}$ année d'école primaire. En dernière année de maternelle, les résultats des bilingues aux différentes tâches métalinguistiques sont soit proches 
(e.g. en identification de phonème, de syllabe et de suppression phonémique) soit inférieurs (e.g. en détection de rime et à l'épreuve syntaxique de closure) à celles des monolingues. Mais en $2^{\mathrm{e}}$ année, les bilingues ont rattrapé leur retard, sauf dans le domaine syntaxique où une légère différence subsiste. Ils obtiennent même de meilleurs scores en identification de mots et en rapidité de lecture de mots et de pseudo-mots. Par ailleurs, cette étude permet de rendre compte des similarités et différences en termes de facteurs impliqués en début d'apprentissage de la lecture chez les monolingues et les bilingues. Les résultats révèlent une contribution importante de la conscience phonologique aux compétences en lecture de mots et en compréhension de phrases écrites en anglais langue seconde. En revanche, les connaissances lexicales et la conscience syntaxique ne semblent pas intervenir, contrairement à ce qui apparaît chez les monolingues. Ce point résulte, selon les auteurs, de l'impact important des instructions axées sur le décodage, à l'étape initiale de l'apprentissage. Geva et Yaghoub Zadeh (2006) viennent compléter ces résultats en suggérant que ces deux types d'habiletés pourraient dans certains cas intervenir. Bien que, dans leur étude portant sur des élèves de $2^{\mathrm{e}}$ année, les connaissances syntaxiques et en vocabulaire soient moins développées que chez les monolingues, elles auraient néanmoins atteint un niveau suffisant pour aider les apprenants en langue seconde à lire efficacement (i.e. rapidement et sans erreurs) les mots et les textes, dans la mesure où le matériel linguistique à lire ne dépasse pas leurs connaissances syntaxiques et lexicales actuelles.

Enfin, la recherche de Roy (2005) est à notre connaissance, la seule s'intéressant aux compétences morpho-dérivationnelles en comparant des enfants bilingues et monolingues de $1^{\mathrm{e}}$ et $2^{\mathrm{e}}$ année. Plusieurs épreuves métamorphologiques ont été administrées :

- une tâche de jugement de relation de mots (e.g. « est-ce que peur et peureux sont de la même famille ?»), évaluant les connaissances de la relation morphologique entre les mots.

- une tâche de closure avec choix de dérivés ou pseudodérivés (e.g. compléter la phrase «pourraistu me _ ton livre", par couter, coutable ou couteur), évaluant les connaissances syntaxiques impliquées dans la compréhension du processus de dérivation.

- une tâche de plausibilité lexicale (e.g. qu'est ce qui ressemble le plus à un mot français arrêteur ou arrêtoume?), évaluant la sensibilité à la construction morphologique des mots français et aux contraintes distributionnelles qui s'y appliquent ${ }^{9}$.

Les résultats obtenus révèlent une infériorité des enfants bilingues à l'épreuve de plausibilité lexicale et une absence de différences entre les deux groupes pour ce qui concerne les épreuves de jugement et de closure. Pour Roy (2005), tous les enfants ont ainsi une bonne compréhension de la famille morphologique et connaissent la catégorie grammaticale des suffixes courants. Les bilingues

\footnotetext{
${ }^{9}$ Les contraintes distributionnelles renvoient par exemple aux règles de légalité des combinaisons affixe - base, spécifiant la ou les catégories grammaticales des bases auxquelles les affixes s'accolent.
} 
ont, en revanche, plus de difficultés à repérer la présence de suffixes dans les pseudo-mots et à déterminer si la combinaison base + affixe est légale. Roy (2005) interprète cette infériorité par un contact moins régulier avec le français, ce qui limite l'évolution de la connaissance des morphèmes et les contraintes distributionnelles qui s'appliquent aux règles de construction morphologique.

\section{Synthèse et perspectives}

A première vue, les résultats des recherches présentées précédemment apparaissent contradictoires. Une synthèse des données obtenues sur chacune des dimensions métalinguistiques les plus pertinentes lors de l'acquisition de l'écrit (en l'occurrence, les compétences métaphonologiques, métasyntaxiques et métamorphologiques) s'avère particulièrement nécessaire pour démêler les facteurs pouvant être à l'origine de ces contradictions et aboutir à une interprétation cohérente sur l'évolution des compétences métalinguistiques et leur rôle lors de l'apprentissage de la lecture en contexte bilingue. Il pourra être fait référence à d'autres recherches qui n'ont pas expressément effectué de comparaisons bilingues/monolingues mais qui apportent des éléments déterminants pour comprendre la contribution de ces compétences à la lecture chez les enfants bilingues. Enfin, le courant des études comparatives inter-langues sera spécifiquement abordé car ses investigations contribuent largement au positionnement sur l'apprentissage de la lecture en contexte bilingue et sur les compétences métalinguistiques qui lui sont associées.

\subsection{Développement métaphonologique et lecture en contexte bilingue}

Le tableau 1 offre un aperçu synoptique des recherches ayant étudié la conscience phonologique des bilingues, comparés aux monolingues. Si ces recherches n’ont pas systématiquement observé une supériorité bilingue, l'analyse et le contrôle des aspects phonologiques chez ces populations restent pour le moins préservés ${ }^{10}$ sans forcément faire émerger une habileté particulière.

${ }^{10}$ à l'exception des résultats sur la rime de Lesaux et Siegel (2003) 
Tableau 1 : Récapitulatif des recherches ayant comparé la conscience phonologique de bilingues et de monolingues

\begin{tabular}{|c|c|c|c|c|c|c|}
\hline Auteurs & Caractéristiques de la population & Langues en contact ${ }^{11}$ & $\begin{array}{l}\text { Langue } \\
\text { testée }\end{array}$ & Niveau & Tâches métaphonologiques & $\begin{array}{c}\text { Comparaison bilingues / } \\
\text { monolingues }\end{array}$ \\
\hline Perregaux $(1994,1995)$ & Enfants immigrés en Suisse & $\mathrm{X}$ - français & français & Maternelle & Segmentation phonémique & Supériorité bilingue \\
\hline Campbell \& Sais (1995) & Enfants scolarisés en école bilingue & italien - anglais & anglais & Maternelle & Détection d'intrus phonémique & Supériorité bilingue \\
\hline \multirow{4}{*}{ Eviatar \& Ibrahim (2000) } & \multirow{4}{*}{$\begin{array}{c}\text { Enfants de langue maternelle russe } \\
\text { vivant en Israël }\end{array}$} & \multirow{4}{*}{ russe - hébreu } & \multirow{4}{*}{ hébreu } & \multirow[b]{2}{*}{ Maternelle } & Détection de phonèmes & Supériorité bilingue \\
\hline & & & & & $\begin{array}{l}\text { Suppression phonémique et } \\
\text { syllabique }\end{array}$ & Différence $\mathrm{NS}^{12}$ \\
\hline & & & & \multirow[b]{2}{*}{$1^{\mathrm{e}}$ année } & Détection de phonèmes & Différence NS \\
\hline & & & & & $\begin{array}{c}\text { Suppression phonémique et } \\
\text { syllabique }\end{array}$ & Supériorité bilingue \\
\hline Ibrahim et al. (2007) & $\begin{array}{c}\text { Enfants de langue maternelle russe } \\
\text { vivant en Israël }\end{array}$ & russe - hébreu & hébreu & $1^{\mathrm{e}}$ année & Suppression syllabique & Supériorité bilingue \\
\hline \multirow{3}{*}{ Lesaux \& Siegel (2003) } & \multirow{3}{*}{$\begin{array}{l}\text { Enfants immigrés ou d'origine } \\
\text { immigrée vivant au Canada }\end{array}$} & \multirow{3}{*}{$\mathrm{X}$ - anglais } & \multirow{3}{*}{ anglais } & \multirow[t]{2}{*}{ Maternelle } & $\begin{array}{c}\text { Identification syllabique } \\
\text { phonémique, suppression } \\
\text { phonémique }\end{array}$ & Différence NS \\
\hline & & & & & Détection de rime & Infériorité bilingue \\
\hline & & & & $2^{\mathrm{e}}$ année & $\begin{array}{c}\text { Suppression syllabique et } \\
\text { phonémique }\end{array}$ & Différence NS \\
\hline Chiappe \& Siegel (1999) & $\begin{array}{c}\text { Enfants locuteurs punjabi vivant au } \\
\text { Canada et exposés à l'anglais depuis } \\
2 \text { ans }\end{array}$ & punjabi - anglais & anglais & $1^{\mathrm{e}}$ année & $\begin{array}{l}\text { identification, localisation, } \\
\text { suppression et substitution } \\
\text { phonémique }\end{array}$ & Différence NS \\
\hline Armand (2000) & $\begin{array}{l}\text { Enfants immigrés ou d'origine } \\
\text { immigrée vivant au Québec }\end{array}$ & $\mathrm{X}$ - français & français & $1^{\mathrm{e}}$ année & $\begin{array}{c}\text { détection de rime et suppression de } \\
\text { phonème }\end{array}$ & Différence NS \\
\hline Demont (2001) & $\begin{array}{c}\begin{array}{c}\text { Enfants scolarisés en école bilingue } \\
\text { à parité horaire }\end{array} \\
\end{array}$ & français - allemand & français & $1^{\mathrm{e}}$ année & Suppression phonémique & Différence NS \\
\hline Demont et al. (2001) & $\begin{array}{c}\begin{array}{c}\text { Enfants scolarisés en école bilingue } \\
\text { ou internationale }\end{array} \\
\end{array}$ & français - allemand & français & $1^{\mathrm{e}}$ année & Suppression phonémique & Différence NS \\
\hline $\begin{array}{l}\text { Geva \& Yaghoub Zadeh } \\
(2006)\end{array}$ & $\begin{array}{l}\text { Enfants immigrés ou d'origine } \\
\text { immigrée vivant au Canada }\end{array}$ & $\mathrm{X}$ - anglais & anglais & $2^{\mathrm{e}}$ année & $\begin{array}{c}\text { Suppression syllabique et } \\
\text { phonémique }\end{array}$ & Différence NS \\
\hline \multirow{3}{*}{ Laurent \& Martinot (2009) } & \multirow{3}{*}{ Enfants scolarisés en école bilingue } & \multirow{3}{*}{ français - occitan } & \multirow{3}{*}{ français } & $3^{\mathrm{e}}$ année & $\begin{array}{l}\text { Suppression et permutation } \\
\text { syllabique et phonémique }\end{array}$ & Différence NS \\
\hline & & & & $4^{\mathrm{e}}$ année & $\begin{array}{l}\text { Permutation syllabique et } \\
\text { phonémique }\end{array}$ & Supériorité bilingue \\
\hline & & & & $5^{\mathrm{e}}$ année & $\begin{array}{c}\text { Permutation syllabique, permutation } \\
\text { et suppression phonémique }\end{array}$ & Supériorité bilingue \\
\hline
\end{tabular}

\footnotetext{
${ }^{11}$ La lettre $\mathrm{X}$ représente une diversité de langues premières des participants.

${ }^{12} \mathrm{NS}$ : non significative

${ }^{13}$ Dans cette étude, l'enseignement en école bilingue correspond à un enseignement à parité horaire (13 heures de Français, 13 heures d'allemand) et en école internationale à un enseignement effectué majoritairement en français.
} 
Tout d'abord, il convient de souligner que les auteurs n'ont pas systématiquement recours aux mêmes tâches et n'étudient pas des unités phonologiques de même taille, si bien que le degré de difficulté des épreuves employées n'est pas toujours identique d'une étude à l'autre et limite les possibilités d'établir des liens cohérents entre certains résultats. Par exemple, Eviatar et Ibrahim (2000) ne distinguent pas les scores de suppression de phonèmes et de syllabes alors que cette dernière unité est, dans plusieurs langues, plus facilement manipulable (e.g. Duncan, Colé, Seymour \& Magnan, 2006). En outre, dans cette recherche, de même que dans celle d'Ibrahim et al. (2007), il n'est pas précisé si l'apprentissage de la lecture a été amorcé en hébreu. Pourtant ce facteur semble constituer un élément déterminant pour comprendre l'évolution des compétences métaphonologiques des bilingues comparée à celle des monolingues.

En effet, en dépit des remarques méthodologiques précédentes, il semble possible de constater que l'avantage métaphonologique (et en particulier métaphonémique) des bilingues est spécifique à certains niveaux scolaires, en particulier en maternelle (Campbell \& Sais, 1995 ; Eviatar \& Ibrahim, 2000 ; Perregaux, 1994, 1995) puis à partir de la $4^{\mathrm{e}}$ année (Laurent $\&$ Martinot, 2009). De telles observations suggèrent que la précocité phonologique bilingue observée en maternelle disparaît lorsque l'apprentissage de la lecture commence. L'introduction d'un apprentissage formel de la lecture semble en effet aboutir à une égalisation des expériences et fait disparaître l'avantage initial des bilingues ( $c f$. Bialystok, 2002). Cette hypothèse rejoint les conclusions de Demont (2001) rappelant que l'enseignement de l'écrit débute généralement, en contexte bilingue comme en contexte monolingue, par un enseignement des correspondances graphèmes - phonèmes et un travail d'analyse de la structure phonologique des mots. Les résultats de Loizou et Stuart (2003) comparant des bilingues (grec-anglais ou anglais-grec) de 5 ans ayant ou non débuté l'apprentissage de l'écrit vont dans le même sens. Les bilingues lecteurs-débutants ont en effet un niveau de conscience phonémique plus développé que celui des bilingues pré-lecteurs. Cette explication est cohérente avec les résultats de Lesaux et Siegel (2003) d'une absence de différence sur la dimension métaphonémique entre bilingues et monolingues de maternelle puisque l'école fréquentée par ces enfants a mis en place un programme d'acquisition précoce des habiletés associées à la lecture telle que la conscience phonologique.

D'autre part, le « retour » de la supériorité bilingue, observé par Laurent et Martinot (2009) après plusieurs années de contact avec l'écrit, renvoie à l'intervention d'un autre facteur à une étape ultérieure de l'apprentissage. Laurent et Martinot (2009) avaient expliqué cet avantage tardif par l'hypothèse d'un niveau seuil de compétences atteint dans les deux langues pour que les effets bénéfiques du bilinguisme puissent se faire sentir. Compte tenu du niveau scolaire où cet avantage apparaît, il pourrait plus précisément être postulé que les compétences concernées par ce niveau seuil soient finalement l'ensemble des habiletés de lecture acquises après plusieurs années 
d'apprentissage. Cette perspective est aussi envisagée par Bialystok (2002) qui suggère que l'effet du bilinguisme serait relativement mineur pendant la période où l'enfant développe ses premières habiletés en lecture et émerge à nouveau lorsque l'enfant atteint un niveau d'efficience dans cette activité. Cependant, d'autres recherches doivent être menées pour éprouver ce postulat et rendre compte du développement métaphonologique en contexte bilingue d'avant l'apprentissage de la lecture jusqu'à la maîtrise du code écrit des langues. Rappelons également que la recherche de Laurent et Martinot (2009) a été menée en langue française, la langue première des bilingues. D'autres données confirmatoires sont ainsi nécessaires dans la langue seconde des enfants.

Ainsi les remarques précédentes suggèrent d'une part que d'autres facteurs tels que les instructions formelles auraient une plus grande importance que le bilinguisme sur le développement métaphonologique, et d'autre part, qu'à long terme, le bilinguisme n'est pas systématiquement un facteur facilitateur du développement métaphonologique mais doit vraisemblablement faire intervenir d'autres éléments tels que l'efficience en lecture pour pouvoir rendre compte de bénéfices durables et stables.

Quoiqu'il en soit, et même chez des enfants bilingues dont l'environnement linguistique ou le contexte socio-économique en font des apprenants à risques (enfants immigrés ou d'origine immigrée dont la langue maternelle, minoritaire dans le pays d'accueil, est dévalorisée, enfants de milieux défavorisés, etc.), les habiletés métaphonologiques ne constituent pas un domaine de compétences affecté par d'éventuels effets négatifs du bilinguisme (Lesaux \& Siegel, 2003). Ces compétences, nécessaires à l'acquisition de la lecture, en langue maternelle comme en langue seconde (Geva \& Yaghoub Zadeh, 2006 ; Lefrançois \& Armand, 2003 ; Lesaux \& Siegel, 2003 ; Siegel, 1993) peuvent même s'avérer particulièrement déterminantes lors de l'apprentissage de la lecture en contexte bilingue. Par exemple, les résultats de nombreuses recherches suggèrent que les enfants dont l'exposition à la langue seconde est limitée (par exemple car la langue de scolarisation n'est pas la langue parlé à la maison) parviennent à un niveau de lecture au moins comparable à celui des monolingues (Chiappe \& Siegel, 1999 ; Da Fontoura \& Siegel, 1995 ; Eviatar \& Ibrahim, 2000 ; Ibrahim et al., 2007 ; Lesaux \& Siegel, 2003 ; Perregaux, 1994, 1995). Ainsi, plusieurs auteurs avancent que la conscience phonologique bien développée des bilingues pourrait permettre de compenser leurs faiblesses au niveau de leurs connaissances langagières en langue seconde (Armand, 2000 ; Ibrahim et al., 2007 ; Lesaux \& Siegel, 2003 ; Perregaux, 1994, 1995). Cependant les données d'autres recherches tendent à montrer des difficultés lors de la lecture en langue seconde par des enfants de minorités linguistiques (August \& Hakuta, 1997, cités par Bialystok, 2002 ; Droop \& Verhoeven, 2003 ; Verhoeven, 1999, 2000), notamment sur la dimension de compréhension, du fait du manque de maîtrise du langage oral (e.g. Droop \& Verhoeven, 2003). 


\subsection{Développement métasyntaxique et lecture en contexte bilingue}

Dans le domaine de la conscience syntaxique, les résultats recueillis par les études précédentes sont hétérogènes ( $c f$. Tableau 2). Néanmoins, une analyse méthodologique des processus spécifiquement impliqués dans les tâches peut permettre de relever plusieurs facteurs de variations. 
Tableau 2 : Récapitulatif des recherches ayant comparé la conscience syntaxique de bilingues et de monolingues

\begin{tabular}{|c|c|c|c|c|c|c|}
\hline Auteurs & Caractéristiques de la population & Langues en contact ${ }^{14}$ & $\begin{array}{l}\text { Langue } \\
\text { testée }\end{array}$ & Niveau & Tâches métasyntaxiques & $\begin{array}{c}\text { Comparaison bilingues / } \\
\text { monolingues }\end{array}$ \\
\hline \multirow{2}{*}{ Cromdal (1999) } & \multirow{2}{*}{$\begin{array}{c}\text { Enfants partiellement (PB) ou } \\
\text { hautement bilingues (HB) scolarisés } \\
\text { en école bilingue. }\end{array}$} & \multirow{2}{*}{ anglais - suédois } & \multirow{2}{*}{ suédois } & \multirow{2}{*}{ Préscolaire } & $\begin{array}{l}\text { Jugement d'agrammaticalité } \\
\text { (phrases asémantiques) }\end{array}$ & $\begin{array}{c}\text { Supériorité bilingue (PB et } \\
\mathrm{HB})\end{array}$ \\
\hline & & & & & $\begin{array}{c}\text { Jugement et correction } \\
\text { d'agrammaticalité }\end{array}$ & Supériorité bilingue (HB) \\
\hline \multirow[b]{2}{*}{ Bialystok (1986) } & \multirow[b]{2}{*}{ Enfants scolarisés en école bilingue } & \multirow[b]{2}{*}{$\mathrm{X}$ - anglais } & \multirow[b]{2}{*}{ anglais } & \multirow[b]{2}{*}{$\begin{array}{c}\text { Maternelle, } 1^{\mathrm{e}} \\
\text { et } 3^{\mathrm{e}} \text { année }\end{array}$} & $\begin{array}{l}\text { Jugement de grammaticalité } \\
\left(\text { phrases } \mathrm{Gs}^{15} \text { ) }\right.\end{array}$ & Supériorité bilingue \\
\hline & & & & & $\begin{array}{l}\text { Jugement d'agrammaticalité } \\
\left(\text { phrases Gs }{ }^{15}\right) \text { Jugement et } \\
\text { correction d'agrammaticalité } \\
\left(\text { phrases gs }{ }^{15}\right)\end{array}$ & Différence NS \\
\hline Bialystok (1988) & $\begin{array}{c}\text { Enfants partiellement (PB) ou } \\
\text { hautement bilingues (HB) }\end{array}$ & anglais - français & anglais & $1^{\mathrm{e}}$ année & Correction d'agrammaticalité & Supériorité bilingue (HB) \\
\hline \multirow{3}{*}{$\begin{array}{l}\text { Galambos \& Goldin- } \\
\text { Meadow (1990) }\end{array}$} & \multirow{3}{*}{$\begin{array}{c}\text { Enfants hautement bilingues } \\
\text { scolarisés dans une école américaine } \\
\text { du Salvador }\end{array}$} & \multirow{3}{*}{ espagnol - anglais } & \multirow{3}{*}{$\begin{array}{l}\text { anglais et } \\
\text { espagnol }\end{array}$} & \multirow{3}{*}{$\begin{array}{c}\text { Préscolaire, } \\
\text { maternelle et } \\
1^{\mathrm{e}} \text { année }\end{array}$} & Jugement d'agrammaticalité & $\begin{array}{c}\text { Supériorité bilingue en } \\
\text { Espagnol }\end{array}$ \\
\hline & & & & & Correction d'agrammaticalité & $\begin{array}{l}\text { Supériorité bilingue au } \\
\text { niveau préscolaire }\end{array}$ \\
\hline & & & & & Explicitation d'agrammaticalité & Différence NS \\
\hline Demont (2001) & $\begin{array}{c}\text { Enfants scolarisés en école bilingue } \\
\text { à parité horaire }\end{array}$ & français - allemand & français & $1^{\mathrm{e}}$ année & $\begin{array}{c}\text { Jugement et correction } \\
\text { d'agrammaticalité ; correction } \\
\text { d'agrammaticalité (phrases } \\
\text { asémantiques) }\end{array}$ & Supériorité bilingue \\
\hline Demont et al. (2001) & $\begin{array}{c}\text { Enfants scolarisés en école bilingue } \\
\text { (EB) ou internationale (EI) }\end{array}$ & français - allemand & français & $1^{\mathrm{e}}$ année & Jugement d'agrammaticalité & $\begin{array}{c}\text { Infériorité bilingue (EI) ou } \\
\text { différence NS (EB) }\end{array}$ \\
\hline Lesaux \& Siegel (2003) & $\begin{array}{l}\text { Enfants immigrés ou d'origine } \\
\text { immigrée vivant au Canada }\end{array}$ & $\mathrm{X}$ - anglais & anglais & $\begin{array}{c}\text { Maternelle et } \\
2^{\mathrm{e}} \text { année }\end{array}$ & Closure & Infériorité bilingue \\
\hline Chiappe \& Siegel (1999) & $\begin{array}{c}\text { Enfants locuteurs punjabi exposés à } \\
\text { l'anglais depuis } 2 \text { ans }\end{array}$ & punjabi - anglais & anglais & $1^{\mathrm{e}}$ année & Closure & Infériorité bilingue \\
\hline Armand (2000) & $\begin{array}{l}\text { Enfants immigrés ou d'origine } \\
\text { immigrée vivant au Québec }\end{array}$ & $\mathrm{X}$ - français & français & $1^{\mathrm{e}}$ année & $\begin{array}{l}\text { Jugement et correction } \\
\text { d'agrammaticalité }\end{array}$ & Infériorité bilingue \\
\hline $\begin{array}{c}\text { Geva \& Yaghoub Zadeh } \\
(2006)\end{array}$ & $\begin{array}{l}\text { Enfants immigrés ou d'origine } \\
\text { immigrée vivant au Canada }\end{array}$ & $\mathrm{X}$ - anglais & anglais & $2^{\mathrm{e}}$ année & Jugement d'agrammaticalité & Infériorité bilingue \\
\hline $\begin{array}{c}\text { Da Fontoura \& Siegel } \\
(1995)\end{array}$ & $\begin{array}{l}\text { Enfants lusophones d'origine } \\
\text { immigrée vivant au Canada }\end{array}$ & portugais - anglais & anglais & $4,5^{\mathrm{e}}, 6^{\mathrm{e}}$ année & Closure & Infériorité bilingue \\
\hline
\end{tabular}

\footnotetext{
${ }^{14}$ La lettre $\mathrm{X}$ représente une diversité de langues premières des participants.

${ }^{15}$ Phrases « Gs » : grammaticales asémantiques. « gS »: agrammaticales sémantiques. " gs » : agrammaticales asémantiques.
} 
Premièrement, à l'instar des positions théoriques de Bialystok (1991, 1993, 1999, 2001), l'avantage des bilingues observé dans plusieurs études (Bialystok, 1986, 1988 ; Cromdal, 1999 ; Demont, 2001 ; Galambos \& Goldin-Meadow, 1990) concernerait plus particulièrement des tâches renvoyant à la dimension de contrôle attentionnel sur les processus linguistiques privilégiant les aspects formels du langage au dépend de la signification (i.e. des tâches où il s'agit de se focaliser sur la grammaticalité d'une phrase indépendamment de sa signification anormale). Les enfants bilingues étant confrontés à des formes linguistiques spécifiques pour exprimer un même concept sémantique, seraient plus à même de représenter les informations formelles et conceptuelles comme des structures séparées ce qui faciliterait la mise en place d'une attention sélective sur les aspects syntaxiques.

Deuxièmement, la conscience syntaxique renvoie également à une connaissance analytique des règles grammaticales. Cette dimension est souvent évaluée par des épreuves de jugement et de correction d'erreurs grammaticales contenues dans des phrases dont l'accès à la signification ne constitue pas un élément parasitant exigeant un haut niveau de contrôle (Bialystok, 1991, 1993, 1999, 2001). Sur cette dimension d'analyse, les effets du bilinguisme semblent principalement dépendre du degré de bilinguisme, ou plus exactement de l'efficience verbale dans la langue considérée. En effet, la supériorité des bilingues à juger et corriger les erreurs syntaxiques apparaît en priorité chez les enfants hautement bilingues (Bialystok, 1988; Cromdal, 1999) ou dans la langue, par exemple la langue première, pour laquelle leurs compétences langagières sont équivalentes à celle des monolingues (Demont, 2001 ; Galambos \& Goldin-Meadow, 1990) ${ }^{16}$. En revanche, les recherches étudiant des bilingues consécutifs en situation d'apprentissage en langue seconde $^{17}$ font généralement apparaître une infériorité par rapport aux locuteurs natifs monolingues à des épreuves de jugement et de correction d'agrammaticalité (Armand, 2000 ; Geva \& Yaghoub Zadeh, 2006) mais aussi à des tâches de closure (Chiappe \& Siegel, 1999 ; Da Fontoura \& Siegel, 1995 ; Lesaux \& Siegel, 2003). Cette difficulté doit être mise en relation avec leurs faibles compétences langagières. En effet, les instruments de mesures employés pour évaluer le niveau d'analyse métasyntaxique exigent des connaissances lexicales importantes. En particulier, les tâches de closure relèvent tout autant de la gestion des contraintes syntaxiques que sémantiques (Layton, Robinson \& Lawson, 1998). Ainsi, chez certains apprenants bilingues, le manque d'exposition à la langue et à sa complexité syntaxique affecte les habiletés métasyntaxiques du fait de compétences

\footnotetext{
${ }^{16}$ Seuls les résultats de l'étude de Demont et al. (2001) font exception à cette interprétation et s'opposent à celle de Demont (2001) réalisée auprès d'une même population. Si les auteurs n'apportent pas d'explications quant aux différences constatées entre ces groupes bilingues scolarisés dans des écoles pourtant comparables, il convient de noter que la taille des échantillons, le nombre d'items pour certaines épreuves, et la période d'expérimentation diffèrent, ce qui pourrait être à l'origine de ces écarts.

17 Notamment des enfants immigrés ou d'origine immigrée dont la langue maternelle est différente de la langue du pays d'accueil dans laquelle ils sont scolarisés.
} 
langagières, notamment en vocabulaire, plus limitées (Armand, 2000 ; Chiappe et Siegel, 1999 ; Da Fontoura et Siegel, 1995 ; Lesaux et Siegel, 2003).

Ainsi, le bilinguisme pourrait influencer différemment certaines dimensions du développement métasyntaxique (davantage la dimension de contrôle que les aspects d'analyse) et, pour ce qui concerne en particulier l'analyse métasyntaxique, seulement dans certaines conditions. Cette analyse serait notamment largement dépendante des compétences langagières. Encore une fois, il ne semble donc pas possible d'affirmer un effet systématique et généralisable du bilinguisme sur le développement métasyntaxique.

De façon similaire, le rôle que peut jouer la conscience syntaxique lors de l'apprentissage de la lecture en contexte bilingue s'avère différent en fonction du degré de bilinguisme. Par exemple, Demont (2001) observe, dans la langue première des bilingues, une médiatisation des habiletés métasyntaxiques à la lecture de mots. En revanche, chez des bilingues immigrés ou d'origine immigrée, les compétences métasyntaxiques en langue seconde semblent trop faibles pour pouvoir intervenir les premières années d'apprentissage (Armand, 2000 ; Lesaux \& Siegel, 2003). Toutefois, lorsque ces compétences atteignent un niveau d'efficience suffisant, leur contribution pourrait s'avérer substantielle (Da Fontoura \& Siegel, 1995), notamment sur les processus de lecture de haut niveau (e.g. lecture rapide ou compréhension de textes, cf. Geva \& Yaghoub Zadeh, 2006 et Lefrançois \& Armand, 2003).

\subsection{Développement métamorphologique et lecture en contexte bilingue}

Concernant la conscience morphologique, les études comparant les performances de bilingues à celles de monolingues sont particulièrement limitées. Alors que Campbel et Sais (1995) ont suggéré un avantage des bilingues italiens-anglais de maternelle à manipuler la structure de mots morphologiquement composés, Roy (2005) avance, pour sa part, une absence de différence entre monolingues et bilingues de primaire, voire même une faiblesse de ces derniers en ce qui concerne les connaissances de la morphologie dérivationnelle. Entre ces deux études, l'âge des enfants, leur langue première, les épreuves utilisées pour les évaluer et le type de morphologie diffèrent, ce qui invite à ne tirer aucune conclusion quant aux origines pouvant expliquer les divergences de résultats.

En outre, si, à notre connaissance, aucune recherche n'a tenté de comparer la contribution de ces compétences à la lecture chez des bilingues et des monolingues, les travaux de Deacon, WadeWooley et Kirby (2007) et de Ramirez, Chen, Geva et Kiefer (2009) apportent des données suggérant l'intervention de la morphologie lors de la lecture dans une autre langue que la langue maternelle.

L'objectif de Deacon et al. (2007) était d'examiner les contributions intra et interlangue de la conscience morpho-syntaxique au développement de la lecture de la $1^{\mathrm{e}}$ à la $3^{\mathrm{e}}$ année chez des 
enfants apprenant à lire en anglais (leur langue maternelle) et en français (programme d'immersion). Les résultats de leur recherche montrent, entre autres, que la conscience morphosyntaxique (évaluée par une tâche de production analogique qui nécessite d'effectuer dans une nouvelle phrase - e.g. je chante des chansons-, la même transformation qu'entre une paire de phrases donnée - e.g. je connais ce garçon / j'ai connu ce garçon-) contribue à la lecture de mots dans les deux langues, après avoir contrôlé l'effet de plusieurs variables (conscience phonologique, vocabulaire et intelligence non verbale). Toutefois, en anglais langue première ce lien concerne davantage la conscience morphologique de $1^{\mathrm{e}}$ année et tend à diminuer alors qu'en français langue seconde, ce sont les dernières mesures $\left(2^{\mathrm{e}}\right.$ et $3^{\mathrm{e}}$ année) de la conscience morphologique qui entretiennent les liens les plus forts avec la lecture. Les auteurs expliquent le décalage et l'augmentation de la contribution de la morphologie pour lire en langue seconde en invoquant le fait qu'un niveau suffisant d'efficience, notamment en vocabulaire, doit être atteint pour que cette contribution se réalise pleinement. Cependant, ils indiquent que certains aspects méthodologiques (e.g. la faiblesse des coefficients de fiabilité des tâches morphologiques) invitent à considérer cette interprétation avec précaution.

Dans le même objectif et après contrôle des mêmes variables associées à la lecture, la recherche de Ramirez et al. (2009) montrent, chez des apprenants hispanophones de $4^{\mathrm{e}}$ et $7^{\mathrm{e}}$ année vivant au Canada depuis plusieurs années (entre 2 et 14 ans, moyenne 8 ;4 ans), que la conscience morphodérivationnelle (évaluée par des épreuves de choix ou de production de mots dérivés pour compléter une phrase) contribue à expliquer le niveau de lecture de mots en anglais langue seconde, mais toutefois de façon moins importante qu'en espagnol langue première, probablement car le système morphologique est plus complexe en espagnol qu'en anglais.

En conclusion, bien qu'il soit prématuré d'affirmer avec précision le rôle des compétences métamorphologiques sur la lecture en contexte bilingue, tout porte à croire que cette dimension est importante, comme cela à été observé chez les monolingues de différentes langues (Abu-Rabia, Share \& Mansour, 2003 ; Carslisle, 2000 ; Casalis \& Louis Alexandre, 2000 ; Lyster, 2002 ; MarecBreton, Gombert \& Colé, 2005 ; Schiff \& Ravid, 2004 ; pour une revue voir Kuo \& Anderson, 2006). Cependant, son intervention pourrait, là encore, interagir avec d'autres facteurs tels que le développement du vocabulaire ou les caractéristiques morphologiques des langues.

\subsection{Les relations entre langues première et seconde et l'impact des caractéristiques linguistiques des langues}

Outre les explications et interprétations envisagées précédemment, force est de constater que plusieurs des recherches citées intègrent, dans un même groupe bilingue, des apprenants de langue maternelle très disparate. Or, pour approfondir les questions abordées dans cette méta-analyse, le 
champ de recherche axé sur les relations entre les langues première et seconde des bilingues ou encore celui prenant en compte les caractéristiques linguistiques de ces langues s'avèrent particulièrement déterminantes et en pleine expansion.

L'impact des expériences de l'écrit en langue première renvoie à la relation d'interdépendance entre langue première et seconde et à la question des processus communs ou spécifiques aux langues. Ce champ d'investigation a donné lieu à plusieurs modélisations théoriques (e.g. Geva, Wade-Wolley \& Shany, 1997 ; Geva \& Siegel, 2000 ; Khan-Horwitz, Shimron \& Spark, 2005, 2006 ; Spark \& Ganschow, 1993), l'hypothèse de l'interdépendance développementale de Cummins (1987, 1989, 1991) étant particulièrement citée dans les recherches s'intéressant aux phénomènes de transfert des compétences entre les langues en contact chez les apprenants bilingues (cross-language transfert). Ainsi, de nombreuses recherches mesurant les compétences métalinguistiques et en lecture, dans les deux langues connues des enfants, ont observé une corrélation importante entre langue première et seconde dans le domaine de la conscience phonologique et de la reconnaissance des mots écrits, ou encore une contribution de la conscience phonologique en langue première sur la lecture en langue seconde, même entre des langues ne partageant pas le même système d'écriture (Gottardo, Yan, Siegel \& Wade-Wooley, 2001 ; Lafrance \& Gottardo, 2005 ; Leikin, Schwartz \& Share, 2009 ; McBride-Chang, Cheung, Chow, Cho \& Choi, 2006 ; Sun-Alperin \& Wang, 2009 ; Verhoven, 2007 ; Wang, Park \& Lee, 2006b). Quelques recherches ont également éprouvé le transfert des compétences métasyntaxiques, et métamorphologiques (Bindman, 2004 ; Deacon et al., 2007 ; Durgunoglu, Mir \& Arino-Marti, 2002 ; Khan-Horwitz et al., 2005 ; Wang, Cheng \& Chen, 2006a) mais les données restent encore insuffisantes pour affirmer clairement l'existence de compétences universelles dans ces domaines. Saiegh-Haddad \& Geva (2008), auprès d'un échantillon de bilingues anglais-arabe de $3^{\mathrm{e}}, 4^{\mathrm{e}}, 5^{\mathrm{e}}$ et $6^{\mathrm{e}}$ année, n'ont d'ailleurs pas observé de corrélations entre la conscience morphologique en anglais langue première et en arabe langue seconde.

Par ailleurs, les relations entre langues première et seconde ne seraient pas unidirectionnelles (Comeau, Cormier, Grandmaison \& Lacroix, 1999) ni l'ordre séquentiel des acquisitions dans les deux langues fixe et stable (Bialystok, 2002). En d'autres termes et de façon réversible, la progression dans une langue influencerait, à certains moments de l'apprentissage, la progression dans l'autre. Les résultats de l'étude longitudinale de Deacon et al. (2007) auprès de bilingues anglais-français vont dans ce sens. Ils montrent en effet, la première année, que la conscience morphosyntaxique en anglais contribue à la lecture dans les deux langues alors que la conscience morphosyntaxique en français ne contribue qu'à la lecture en français. La troisième année, ces dernières participent à la lecture en français et en anglais. Egalement, dans une étude comparant des bilingues anglais-chinois équilibrés et des apprenants sinophones débutant l'anglais langue seconde, de maternelle et de $1^{\mathrm{e}}$ année, Bialystok et al. (2005b) observent un transfert de la conscience 
phonologique pour les deux groupes alors que le transfert des stratégies de lecture n'est mis en place que chez apprenants en langue seconde. D'autres données suggèrent également que, tout au moins lorsque les principes d'écriture des langues sont éloignés, la référence aux stratégies de la langue première, renforcée par les instructions, concernerait surtout les stades élémentaires de l'apprentissage de la lecture en langue seconde. Enfin, d'autres études suggèrent que la réversibilité $\mathrm{du}$ transfert n'est pas systématique et pourrait aussi dépendre des caractéristiques des langues (Ramirez et al., 2009 ; Wang et al., 2006a ; Saiegh-Haddad \& Geva, 2008).

La perspective crosslinguistique, qui s'attache à analyser les différences entre des groupes bilingues de différentes langues premières, permet de nuancer et approfondir les premières conclusions sur le transfert inter-langue en accordant une place centrale aux caractéristiques des langues premières et à leur rapport de proximité avec la langue seconde. Les études développées dans cette perspective se sont surtout intéressées à des populations adultes (e.g. Fender, 2003 ; Wang, Koda \& Perfetti, 2003 ; cf. Koda, 2005, 2007 pour une revue). Mais quelques données recueillies auprès d'enfants apportent des éléments de compréhension sur les variations inter-groupes quant au développement de certaines compétences métalinguistiques et aux relations entre les deux langues connues des enfants bilingues. Par exemple, Bialystok, Majumder et Martin (2003) ont observé que les bilingues espagnol-anglais de $1^{\mathrm{e}}$ et $2^{\mathrm{e}}$ année, sont plus performants que leurs pairs monolingues de langue anglaise à une tâche de segmentation phonémique alors que les bilingues chinois-anglais sont moins performants que ces derniers. La plus grande similarité des structures phonologiques entre l'espagnol et l'anglais, et éventuellement la facilité d'accéder à une conscience phonologique en espagnol ${ }^{18}$ permettent d'expliquer que l'analyse phonologique en anglais soit plus facilement menée par les bilingues de langue première espagnol.

De même, Bialystok, Luk et Kwan (2005a) ont comparé des groupes d'enfants bilingues de $1^{\mathrm{e}}$ année dont la langue de scolarisation est l'anglais et la langue familiale est soit le chinois, soit l'hébreu, soit l'espagnol, ces langues faisant également l'objet d'un enseignement de la lecture. Leurs résultats montrent, tout d'abord, de moins bonnes performances en décodage et en segmentation phonémique en anglais chez les enfants chinois. Par ailleurs, la corrélation, observée entre le décodage dans les deux langues, est non significative pour le groupe chinois, mais élevée pour les groupes hébreu et espagnol. Ainsi, le transfert des stratégies de lecture s'effectuerait mais seulement si les deux langues sont basées sur le même principe alphabétique.

Toujours sur la dimension métaphonologique, mais à l'aide de comparaisons entre l'anglais et le grec, Loizou et Stuart (2003) ont montré une supériorité des bilingues anglais-grec de 5 ans par rapport à leurs pairs monolingues anglophones sur plusieurs tâches métaphonologiques

\footnotetext{
${ }^{18}$ car cette langue utilise un système phonétique simple.
} 
(suppression de syllabe et de phonème, identification phonémique etc.). En revanche, les bilingues grec-anglais de même âge obtiennent des scores moins élevés que leur pair monolingues grécophones. L'avantage bilingue pourrait donc dépendre de la complexité phonologique des langues. Si le système phonologique de la langue première est plus complexe que celui de la langue seconde, comme cela est le cas pour les bilingues anglais-grec, le développement métaphonologique serait facilité, alors que le cas de figure inverse (un système phonologique moins complexe dans la langue première que dans la langue seconde) n'apporterait pas de bénéfices précoces.

Cependant, les recherches inter-langues premières chez les enfants bilingues restent très circonscrites aux habiletés métaphonologiques. De futures études suivant cette méthodologie doivent se développer sur d'autres dimensions métalinguistiques et en comparant un panel plus important de langues premières et secondes.

Quoiqu'il en soit, les caractéristiques des langues peuvent expliquer certaines données indiquant une supériorité ou au contraire un déficit bilingue sur des unités phonologiques particulières plus ou moins saillantes dans les systèmes écrits (e.g. Bruck \& Genesee, 1995 ; Saiegh-Haddad, Kogan \& Walters, 2009). Par exemple, les résultats faisant apparaître une infériorité bilingue concernant la rime chez des prélecteurs bilingues ayant l'anglais comme langue seconde (Lesaux \& Siegel, 2003) peuvent être expliqués par le fait que cette unité est particulièrement pertinente en langue anglaise mais pas forcément dans toutes les langues (Goswami, Ziegler \& Richardson, 2005).

Ainsi, les relations entre les langues connues des apprenants, la façon dont elles sont mises en place en fonction des caractéristiques des langues et la façon dont elles évoluent au cours des apprentissages méritent d'être prises en compte avec plus de précisions. De plus, la dimension linguistique étudiée et le mode d'évaluation des compétences s'avèrent particulièrement déterminants pour comprendre conjointement l'intervention des processus cognitifs généraux et des connaissances spécifiques aux langues au cours du développement métalinguistique. Ce champ de recherche reste donc à approfondir.

\section{Conclusion}

A l'issue de cette synthèse, il semble que le bilinguisme ne soit pas à lui seul un facteur affectant de façon univoque le développement des compétences métalinguistiques associées à la lecture. Comme le suggère Bruck et Genesee (1995), le bilinguisme aurait un effet plus sélectif qu'universel sur le développement métalinguistique, effet variable en fonction du domaine étudié, des conditions d'apprentissage de la lecture ou des compétences langagières.

En outre, les compétences orales ne sont généralement pas une habileté privilégiée chez les enfants bilingues. On retrouve même un vocabulaire généralement moins étendu chez les bilingues (Armand, 2000 ; Cobo-Lewis, Pearson, Eilers \& Imbel, 2002 ; Geva \& Yaghoub Zadeh, 2006 ; 
Lesaux et Siegel 2003), ce déficit pouvant compromettre l'apprentissage de la lecture (Geva \& Yaghoub Zadeh, 2006 ; Proctor, Carlo, August \& Snow, 2005 voir également Bialystok, 2002, 2005) notamment la compréhension en lecture (Droop et Verhoven 2003 ; Carlisle, Beeman, Davis \& Spharim, 1999).

En revanche, Bialystok et ses collaborateurs (Bialystok, 1997 ; Bialystok, Shenfield \& Codd, 2000) ont montré que le développement des concepts sur l'écrit était positivement influencé par le bilinguisme. Les enfants bilingues semblent en effet particulièrement conscients de l'invariabilité de l'écrit pour représenter une forme symbolique et des modifications des correspondances formeréférent entre les systèmes des diverses langues écrites. La compréhension de ces concepts est sans conteste, une condition préalable à la lecture. Ainsi, les effets positifs les plus généralement visibles du bilinguisme dans le domaine de l'apprentissage de la lecture sont relatifs à l'établissement de ces concepts sur l'écriture, son caractère invariable mais arbitraire.

Malgré ces spécificités, l'acquisition de la lecture en contexte bilingue n'apparaît pas fondamentalement différente de l'acquisition de la lecture dans une seule langue dans le sens où l'on retrouve l'intervention des mêmes variables et des mêmes procédures (Chiappe \& Siegel, 1999 ; Da Fontoura \& Siegel, 2000 ; Geva \& Yaghoub Zadeh, 2006 ; Meschyan \& Hernandez, 2006).

Pour conclure, les éléments développés dans cette synthèse renvoient à des questions didactiques fondamentales et toujours d'actualité sur la façon d'aborder l'apprentissage de l'écrit sur le mode bilingue. Avant de pouvoir contribuer aux débats sur les méthodes d'enseignement de la lecture auprès de ces populations, nous pensons que les caractéristiques des élèves bilingues et des langues doivent être minutieusement étudiées et qu'il faut éviter d'introduire une hiérarchie entre les langues qui se résumerait à dévaloriser l'une des deux. Ainsi, les dispositifs bilingues de transition qui suppriment l'apprentissage en langue première une fois qu'il a rempli son rôle de support aux apprentissages en langue seconde n'ont pas forcément obtenus les effets positifs escomptés ( $c f$. Lucchini, 2002) et invitent à envisager l'apprentissage bilingue des langues écrites sur le long terme. Dans cette perspective, il est tout autant nécessaire de tenir compte des atouts mais aussi des difficultés spécifiques des bilingues pour aboutir à une maîtrise de l'écrit dans les deux langues la plus équilibrée possible. Enfin, si le repérage de certains aspects généraux des effets du bilinguisme peuvent contribuer à comprendre ces avantages et ces faiblesses, les aspects spécifiques tels que les caractéristiques des langues ne doivent pas être minimisés. 


\section{Références bibliographiques :}

Abu-Rabia, S., Share, D., \& Mansour, M. S. (2003). Word recognition and basic cognitive processes among reading-disabled and normal readers in Arabic. Reading and Writing, 16(5), 423-442.

Armand, F. (2000). Le rôle des capacités métalinguistiques et de la compétence langagière orale dans l'apprentissage de la lecture en français langue première et seconde. La revue canadienne des langues vivantes, 56, 470-495.

BenZeev, S. (1977). The influence of bilingualism on cognitive strategy and cognitive development. Child Development, 48, 1009-1018.

Berthoud, A. C. (1982). Activité métalinguistique et acquisition d'une langue seconde; étude des verbes déictiques allemands. Berne: Lang.

Bialystok, E. (1986). Factors in the growth of linguistic awareness. Child Development, 57(2), 498510.

Bialystok, E. (1988). Level of bilingualism and levels of linguistic awareness. Developmental Psychology, 24(4), 560-567.

Bialystok, E. (1991). Metalinguistic dimensions of bilingual language proficiency. In E. Bialystok (Ed.), Language processing in bilingual children. (pp. 113-140). New York, NY, US: Cambridge University Press.

Bialystok, E. (1993). Metalinguistic awareness: The development of children's representations of language. In C. Pratt \& A. F. Garton, (Eds.), Systems of representation in children: development and use. (pp. 213-233). Chichester: John Wiley \& sons.

Bialystok, E. (1997). Effects of bilingualism and biliteracy on children's emerging concepts of print. Developmental Psychology, 33, 429-440.

Bialystok, E. (1999). Cognitive complexity and attentional control in the bilingual mind. Child Development, 70(3), 636-644.

Bialystok, E. (2001). Bilingualism in development: Language, literacy, and cognition. New York, NY, US: Cambridge University Press.

Bialystok, E. (2002). Acquisition of literacy in bilingual children: A framework for research. Language Learning, 52(1), 159-199.

Bialystok, E. (2005). Consequences of bilingualism for cognitive development. In J. F. Kroll \& A. M. B. De Groot (Eds.), Handbook of bilingualism: Psycholinguistic approaches. (pp. 417-432). New York, NY, US: Oxford University Press.

Bialystok, E. (2007). Acquisition of Literacy in Bilingual Children: A Framework for Research. Language learning, 57(1), 45-77.

Bialystok, E., Luk, G., \& Kwan, E. (2005a). Bilingualism, biliteracy, and learning to read: Interactions among languages and writing systems. Scientific Studies of Reading, 9(1), 43-61.

Bialystok, E., Majumder, S., \& Martin, M. M. (2003). Developing phonological awareness: Is there a bilingual advantage? Applied Psycholinguistics, 24(1), 27-44.

Bialystok, E., McBride-Chang, C., \& Luk, G. (2005b). Bilingualism, language proficiency, and learning to read in two writing systems. Journal of Educational Psychology, 97(4), 580-590.

Bialystok, E., Shenfield, T., \& Codd, J. (2000). Languages, scripts, and the environment: Factors in developing concepts of print. Developmental Psychology, 36, 66-76.

Bindman, M. (2004). Grammatical awareness across languages and the role of social context: Evidence from English and Hebrew. In T. Nunes \& P. Bryant (Eds.), Handbook of children's literacy (pp. 691-709). Dordrecht, the Netherlands: Kluwer.

Bruck, M., \& Genesee, F. (1995). Phonological awareness in young second language learners. Journal of Child Language, 22, 307-324.

Bruyninckx, M., \& Harmegnies, B. (1993). Bilinguismes. Revue de Phonétique Appliquée, 108-109, 192-217.

Campbell, R., \& Sais, E. (1995). Accelerated metalinguistic (phonological) awareness in bilingual children. British Journal of Developmental Psychology, 13, 61-68. 
Carlisle, J. F. (1995). Morphological awareness and early reading achievement. In L. B. Feldman (Ed.), Morphological aspects of language processing. (pp. 189-209). Hillsdale, NJ, England: Lawrence Erlbaum Associates, Inc.

Carlisle, J. F. (2000). Awareness of the structure and meaning of morphologically complex words: Impact on reading. Reading and Writing: an Interdisciplinary Journal, 12(3), 169-190.

Carlisle, J. F., \& Nomanbhoy, D. M. (1993). Phonological and morphological awareness in first graders. Applied Psycholinguistics, 14(2), 177-195.

Carlisle, J. F., Beeman, M., Davis, L. H., \& Spharim, G. (1999). Relationship of metalinguistic capabilities and reading achievement for children who are becoming bilingual. Applied Psycholinguistics, 20(4), 459-478.

Casalis, S., \& Louis-Alexandre, M.-F. (2000). Morphological analysis, phonological analysis and learning to read French: A longitudinal study. Reading and Writing: an Interdisciplinary Journal, 12(3), 303-335.

Chiappe, P., \& Siegel, L. S. (1999). Phonological awareness and reading acquisition in English- and Punjabi-speaking Canadian children. Journal of Educational Psychology, 91(1), 20-28.

Cobo-Lewis, A. B., Pearson, P. Z., Eilers, R. E., \& Umbel, V. C. (2002). Effects of bilingualism and bilingual education on oral and written Spanish skills: A multifactor study of standardized test outcomes. In D. K. Oller \& R. E. Eilers (Eds.), Language and literacy in bilingual children (pp. 98117). Clevedon, UK: Multilingual Matters.

Comeau, L., Cormier, P., Grandmaison, A. R., \& Lacroix, D. (1999). A longitudinal study of phonological processing skills in children learning to read in a second language. Journal of Educational Psychology, 91(1), 29-43.

Cromdal, J. (1999). Childhood bilingualism and metalinguistic skills: Analysis and control in young Swedish-English bilinguals. Applied Psycholinguistics, 20, 1-20.

Cummins, J. (1978). Métalinguistic development of children in bilingual education programs: Data from Irish and Canadian Ukrainian-English programs. In M. Paradis (Ed.), Aspects of bilingualism (pp. 127-138). Columbia: Hornbeam Press.

Cummins, J. (1987). L'éducation bilingue: Théorie et mise en oeuvre. In Centre pour la recherche et innovation dans l'enseignement (Ed.), L'éducation multiculturelle (pp. 323-354). Paris: OCDE.

Cummins, J. (1989). Language and literacy acquisition in bilingual contexts. Journal of Multilingual and Multicultural Development, 10(1), 17-31.

Cummins, J. (1991). Interdependence of first-and second-language proficiency in bilingual children. In E. Bialystok (Ed.), Language processing in bilingual children. Cambridge: Cambridge University Press.

Cummins, J., \& Swain, M. (1986). Bilingualism in education. London: Longman.

Da Fontoura, H. A., \& Siegel, L. S. (1995). Reading, syntactic, and working memory skills of bilingual Portuguese-English Canadian children. Reading and Writing: an Interdisciplinary Journal, 7(1), 139-153.

Deacon, H., Wade-Woolley, L. \& Kirby, J. (2007). Crossover: The role of morphological awareness in French immersion children's reading. Developmental Psychology, vol. 43, 3, 732-746.

Demont, E. (2001). Contribution de l'apprentissage précoce d'une deuxième langue au développement de la conscience linguistique et à l'apprentissage de la lecture. Journal International de Psychologie, 36(4), 274-285.

Demont, E., \& Gombert, J. E. (1996). Phonological awareness as a predictor of recoding skills and syntactic awareness as a predictor of comprehension skills. British Journal of Educational Psychology, 66(3), 315-322.

Demont, E., \& Gombert, J. E. (2007). Relations entre conscience phonologique et apprentissage de la lecture: Peut-on sortir de la relation circulaire? In E. Demont \& M. L. Metz-Lutz. (Eds.), L'acquisition du langage et ses troubles (pp. 47-79). Marseille: Solal.

Demont, E., Louvet, E., \& Nocus, I. (2001). L'enseignement bilingue en Alsace: Quelles influences sur l'apprentissage de la lecture \& la conscience linguistique? In E. Regnault (Ed.), Les enjeux du pluralisme linguistique pour les systèmes d'éducation et de formation. Paris: L'Harmattan. 
Droop, M., \& Verhoeven, L. (2003). Language proficiency and reading ability in first- and secondlanguage learners. Reading Research Quarterly, 38(1), 78-103.

Duncan, L. G., Colé, P., Seymour, P. H. K., \& Magnan, A. (2006). Differing sequences of metaphonological development in French and English. Journal of Child Language, 33(2), 369-399.

Durgunoğlu, A. Y., Mir, M., \& Arino-Martí, S. (2002). The relationship between bilingual children's readinc and writing in their two languages. In S; Ransdell \& M. Barbier (Eds), Psycholinguistic approaches to understanding second language writing (pp. 81-100). Dordrecht: Kluwer.

Eviatar, Z., \& Ibrahim, R. (2000). Bilingual is as bilingual does: Metalinguistic abilities of arabicspeaking children. Applied Psycholinguistics, 21(4), 451-471.

Fender, M. (2003). English word recognition and word integration skills of native Arabic- and Japanese-speaking learners of English as a second language. Applied Psycholinguistics, 24(2), 289315.

Galambos, S., \& Goldin-Meadow, S. (1990). The effects of learning two languages on levels of metalinguistic awareness. Cognition, 34(1), 1-56.

Gaux, C., \& Gombert, J. E. (1999b). Implicit and explicit syntactic knowledge and reading in preadolescents. British Journal of Developmental Psychology, 17(2), 169-188.

Geva, E., \& Siegel, L. S. (2000). Orthographic and cognitive factors in the concurrent development of basic reading skills in two languages. Reading and Writing: an Interdisciplinary Journal, 12(1), 1-30.

Geva, E., \& Yaghoub Zadeh, Z. (2006). Reading efficiency in native English-speaking and Englishas-a-second-language children: The role of oral proficiency and underlying cognitive-linguistic processes. Scientific Studies of Reading, 10(1), 31-57.

Geva, E., Wade-Woolley, L., \& Shany, M. (1997). Development of reading efficiency in first and second language. Scientific Studies of Reading, 1(2), 119-144.

Gombert, J. E. (1990). Le développement métalinguistique. Paris: Presses Universitaires de France.

Goswami, U., Ziegler, J. C., \& Richardson, U. (2005). The effects of spelling consistency on phonological awareness: A comparison of English and German. Journal of Experimental Child Psychology, 92(4), 345-365.

Gottardo, A., Yan, B., Siegel, L. S., \& Wade-Woolley, L. (2001). Factors related to English reading performance in children with Chinese as a first language: More evidence of cross-language transfer of phonological processing. Journal of Educational Psychology, 93(3), 530-542.

Ianco-Worall, A. (1972). Bilingualism and cognitive development. Child Development, 43, 13901400.

Ibrahim, R., Eviatar, Z., \& Aharon-Peretz, J. (2007). Metalinguistic awareness and reading performance: A cross language comparison. Journal of Psycholinguistic Research, 36(4), 297-317.

Kahn-Horwitz, J., Shimron, J., \& Sparks, R. L. (2005). Predicting foreign language reading achievement in elementary school students. Reading and Writing: an Interdisciplinary Journal,, 18(6), 527-558.

Kahn-Horwitz, J., Shimron, J., \& Sparks, R. L. (2006). Weak and strong novice readers of english as a foreign language: Effects of first language and socioeconomic status. Annals of Dyslexia, 56(1), 161-185.

Koda, K. (2005). Insights into second language reading: A cross-linguistic approach. Cambridge; New York: Cambridge University Press.

Koda, K. (2007). Reading and language learning: Crosslinguistic constraints on second language reading development. Language Learning, 57(1), 1-44.

Kuo, L.-j., \& Anderson, R. C. (2006). Morphological awareness and learning to read: A crosslanguage perspective. Educational Psychologist, 41(3), 161-180.

Lafrance, A., \& Gottardo, A. (2005). A longitudinal study of phonological processing skills and reading in bilingual children. Applied Psycholinguistics, 26(4), 559-578.

Laurent, A., \& Martinot, C. (2009). Bilingualism and phonological awareness: the case of bilingual (French-Occitan) children. Reading and Writing: an Interdisciplinary Journal, online first: DOI 10.1007/s11145-009-9209-3. 
Layton, A., Robinson, J., \& Lawson, M. (1998). The relationship between syntactic awareness and reading performance. Journal of Research in Reading, 21(1), 5-23.

Lefrançois, P., \& Armand, F. (2003). The role of phonological and syntactic awareness in second language reading: The case of Spanish-speaking learners of French. Reading and Writing: an Interdisciplinary Journal, 16, 219-246.

Leikin, M., Schwartz, M., \& Share, D.L. (2009). General and specific benefits of bilingualism: a Russian-Hebrew study of beginning literacy. Reading and Writing: an Interdisciplinary Journal, online first: DOI 10.1007/s11145-009-9210-x.

Lesaux, N. K., \& Siegel, L. S. (2003). The development of reading in children who speak English as a second language. Developmental Psychology, 39(6), 1005-1019.

Loizou, M., \& Stuart, M. (2003). Phonological awareness in monolingual and bilingual English and Greek five-years-olds. Journal of Research in Reading, 26(1), 3-18.

Lucchini, S. (2002). L'apprentissage de la lecture en langue seconde. La formation d'une langue de référence chez les enfants d'origine immigrée. Cortil-Wodon: Editions Modulaires Européennes.

Lyster, S.-A. H. (2002). The effects of morphological versus phonological awareness training in kindergarten on reading development. Reading and Writing: an Interdisciplinary Journal, 15(34), 261-294.

Mackey, W. F. (1976). Bilinguisme et contact des langues. Paris: Editions Klincksieck.

Marec-Breton, N., Gombert, J.-E., \& Colé, P. (2005). Traitements morphologiques lors de la reconnaissance des mots écrits chez des apprentis lecteurs. L'année Psychologique, 105(1), 9-45.

McBride-Chang, C., Cheung, H., Chow, B. W.-Y., Chow, C.S.L., \& Choi, L. (2006). Metalinguistic skills and vocabulary knowledge in L1 and L2. Reading and Writing: an Interdisciplinary Journal, 19, 695-716.

McLaughlin, B. (1991). Theories of second-language learning. London: Edward Arnold.

Meschyan, G., \& Hernandez, A. E. (2006) Cognitive factors in second-language acquisition and literacy learning. In C. A. Stone, E.R. Silliman, B. J. Ehren, \& K. Apel (Eds.). Handbook of language and literacy: Development and disorders. (pp. 73-81). New York: The Guilford Press.

Palmer, M. (1972). Effects of categorisation, degree of bilingualism and language upon recall of select monolingual and bilinguals. Journal of Educational Psychology, 63, 160-164.

Pearl, E., \& Lambert, W. E. (1962). The relation of bilingualism to intelligence. Psychological Monographs, 76, 1-23.

Perregaux, C. (1994). Les enfants à deux voix. Des effets du bilinguisme sur l'apprentissage de la lecture. Paris: Peter Lang.

Perregaux, C. (1995). Reconnaître les atouts linguistiques des enfants bilingues pré-lecteurs. TRANEL (Travaux neuchâtelois de linguistique), 19, 125-139.

Proctor, C. P., Carlo, M., August, D., \& Snow, C. (2005). Native Spanish-speaking children reading in English: Toward a model of comprehension. Journal of Educational Psychology, 97(2), 246-256.

Ramirez, G., Chen, X., Geva, E. \& Kiefer, H. (2009). Morphological awareness in Spanish-speaking English langue learners: within and cross-language effects on word reading. Reading and Writing: an Interdisciplinary Journal, online first: DOI 10.1007/s11145-009-9203-9.

Rego, L. L., \& Bryant, P. E. (1993). The connection between phonological, syntactic and semantic skills and children's reading and spelling. European Journal of Psychology of Education, 8(3), 235246.

Roy, C. (2005). Comparaison des compétences en morphologie dérivationnelle chez les francophones et non-francophones apprentis lecteurs. Unpublished mémoire en linguistique, Université du Québec, Montréal.

Saiegh-Haddad, E., \& Geva, E. (2008). Morphological awareness, phonological awareness and reading in English-Arabic bilingual children. Reading and Writing: an Interdisciplinary Journal, 21, 481-504.

Saiegh-Haddad, E., Kogan, N., \& Walters, J. (2009). Universal and language-specific constraints on phonemic awareness: evidence from Russian-Hebrew bilingual children. Reading and Writing: an Interdisciplinary Journal, 
Schiff, R., \& Ravid, D. (2004). Vowel representation in written Hebrew: Phonological, orthographic and morphological contexts. Reading and Writing: an Interdisciplinary Journal, 17, 214-265.

Siegel, L. (1993). The development of reading. In H.W. Reese (Ed.), Advances in child development and behavior (pp. 63-97). San Diego, CA: Academic Press.

Sparks, R., \& Ganschow, L. (1993a). Searching for the cognitive locus of foreign language learning difficulties: Linking first and second language learning. Modern Language Journal, 77(3), 289302.

Sun-Alperin, M.K., \& Wang, M. (2009). Cross-language transfer of phonological and orthographic processing skills from Spanish L1 to English L2. Reading and Writing: an Interdisciplinary Journal, online first: DOI 10.1007/s11145-009-9221-7.

Titone, R. (1972). Le bilinguisme précoce. Bruxelles: Delessert.

Verhoeven, L. (1999). Acquisition of reading in second language. Reading Research Quarterly, 25, 90-114.

Verhoeven, L. (2000). Components in early second language reading and spelling. Scientific Studies of Reading, 4, 313-330.

Verhoeven, L. (2007). Early bilingualism, language transfer, and phonological awareness. Applied Psycholinguistics, 28, 425-439.

Vygotsky, L. S. (1934, trad. 1985). Pensée et langage. Paris: Editions sociales.

Wang, M., Cheng, C., \& Chen, S. W. (2006a). Contribution of morphological awareness to ChineseEnglish biliteracy acquisition. Journal of Educational Psychology, 98, 542-553.

Wang, M., Koda, K., \& Perfetti, C. A. (2003). Alphabetic and nonalphabetic L1 effects in English word identification: A comparison of Korean and Chinese English L2 learners. Cognition, 87 (2), 129-149.

Wang, M., Park, Y. J., \& Lee, K. R. (2006b). Korean-English biliteracy acquisition: Cross language and orthography transfer. Journal of Educational Psychology, 98, 148-158. 